Trascender, Contabilidad y Gestión. Vol. 7, Núm. 19 (enero - abril del 2022).

Universidad de Sonora. Departamento de Contabilidad. México.

ISSN: 2448-6388. Reserva de Derechos 04-2015-04172070800-203.

\title{
Diagnóstico del Modelo de Negocios en la industria manufacturera de Ciudad Juárez: "Situación actual sobre su aplicación y características que lo definen"
}

\author{
Diagnosis of the Business Model in the manufacturing industry of Ciudad Juarez: \\ "Current situation on its application and characteristics that define it"
}

Erick Daniel Nava Orihuela ${ }^{1}$; Juan Alfonso Toscano Moctezuma ${ }^{2}$

Recibido: 23 de junio de 2021.

Aceptado: 28 de agosto de 2021.

DOI: https://doi.org/10.36791/tcg.v7i19.131

JEL: M00. Generalidades. Administración de empresas y economía de la empresa.

\section{Resumen}

El presente artículo tiene como objetivo el investigar sobre el estatus actual del Modelo de Negocios en cuanto a su aplicación y características en la industria manufacturera de Ciudad Juárez, por medio de la revisión con métodos cualitativos (entrevistas) y el contraste o empate con la revisión bibliográfica, con el fin de obtener conclusiones útiles para la posible mejora del modelo de negocios en empresas de manufactura. Los resultados de las entrevistas aplicadas a un pequeño grupo de funcionarios directivos y a un experto consultor independiente señalan, que no todos los directivos de las empresas en esta industria tienen claro sobre el concepto de este Modelo de Negocios, ni tampoco cuentan con autonomía para la toma de decisiones, ya que la gran mayoría de estas empresas están controladas por sus oficinas matrices ubicadas en otros países del mundo. Se concluye que el constante monitoreo del Modelo de Negocios y el diagnóstico del mismo ayuda a las grandes empresas de la

\footnotetext{
${ }^{1}$ Erick Daniel Nava Orihuela. Ingeniero en Mecatrónica. Investigador independiente Universidad Autónoma de Ciudad Juárez. Correo: al194532@alumnos.uacj.mx. Correo alterno: navalb52@gmail.com.

2 Juan Alfonso Toscano Moctezuma. Licenciado en Contaduría con Maestría en Administración. Doctor en Contabilidad y Auditoría. Coordinador del Programa de Maestría en Administración de la Universidad Autónoma de Ciudad Juárez, Chihuahua, México. Correo institucional: jtoscano@uacj.mx. Correo alterno: at_educacion@hotmail.com. ORCID: https://orcid.org/0000-0002-8543-3600.
} 
industria manufacturera en Ciudad Juárez a mantener por una parte su vigencia y adaptabilidad, y por la otra que debido a la diversidad de sectores en esta industria manufacturera no se permite la caracterización de un modelo de negocios. Asimismo, existen similitudes en productos y procesos desarrollados en la región, aunque actualmente no tienden ser de alta tecnología, sin embargo, se tiene el potencial en esta industria manufacturera.

Palabras clave: modelos de negocios, propuesta de valor, estructura de costos, segmentos de mercado.

\section{Abstract}

The objective of this paper is to investigate the current status of the Business Model in terms of its application and characteristics in the manufacturing industry of Ciudad Juarez, through the review with qualitative methods (interviews) and the contrast or tie with the review bibliographic, in order to obtain useful conclusions for the possible improvement of the business model in manufacturing companies. The results of the interviews applied to a small group of executive officers and an independent expert consultant indicate that not all executives of companies in this industry are clear about the concept of this Business Model, nor do they have autonomy to make decisions, since the vast majority of these companies are controlled by their head offices located in other countries of the world. It is concluded that the constant monitoring of the Business Model and its diagnosis helps the large companies of the manufacturing industry in Ciudad Juarez to maintain, on the hand, its validity and adaptability, and on the other, due to the diversity of activities in this manufacturing industry the characterization of a business model is not allowed. Likewise, there are similarities in products and processes developed in the region, although currently they do not tend to be high-tech, however, there is potential in this manufacturing industry.

Keywords: business model, value proposition, cost structure, market segments.

\section{Introducción}

El modelo de negocios (Business Model o BM por sus siglas en inglés) es un componente medular de cualquier empresa, ya que es la manera de cómo la organización crea y captura valor, llevando a cabo una conceptualización y materialización del propósito de la misma, teniendo como objetivo ser claro siempre con una estrecha relación con las estrategias del negocio (Zott, Amit, y Lorenzo, 2010: 216-226).

Asimismo, el establecimiento del BM de cualquier empresa funciona como una manera de organizar e interrelacionar las actividades para entregar la propuesta de valor, es decir, llegar con mayor precisión a lo que se dedica la empresa y cómo lo hace desde un punto de vista más holístico (Teece, 2010:172-194). Un BM es entonces el contenido, la estructura y los métodos de las estrategias diseñadas para crear valor mediante la diversa explotación de oportunidades de negocio en cuestión (Zott y Amit, 2010: 1-43).

De este modo se puede conceptualizar al BM como la herramienta o el conjunto de las mismas, las cuales son las bases que sirven para la creación de valor de un negocio, proyecto u organización con fines de lucro. Es un concepto que es cada vez más importante en investigaciones tanto empíricas como las meramente teóricas o académicas, pero que además resultan de vital importancia para el mejoramiento de toda la estructura en una empresa. Es un análisis, que enfatiza en la explicación de un sistema integral (la estructura de la empresa que se compone de la mayor de los sistemas 
periféricos) a través del cual las empresas hacen negocios, en un ambiente de cambio como lo es la realidad actual, incremento de la competencia e incremento de la complejidad del mercado (Morris, 2010: 191-204).

De manera más amplia la función de un BM trata explícitamente de identificar o delimitar el segmento del mercado, especificar el mecanismo de generación de ingresos, definir la estructura de la cadena de valor, describir cada uno de los elementos de la empresa en la cadena de valor y finalmente formular una estrategia competitiva que obtenga y mantenga una ventaja sobre la competencia (Chesbrough, 2010: 354-363). Todo lo anterior con miras a la creación del valor de manera estratégica. Es así como se pueden complementar el concepto del BM y las ideas de distintos autores, profesionales y administradores que tratan con el tema, con la descripción gráfica mostrada en la Figura 1, donde, de manera integral se presentan los diferentes aspectos que debe tratar para acercarse al cumplimiento de su objetivo mayormente consensado.

Figura 1. Mapa conceptual típico del modelo de negocios (BM).

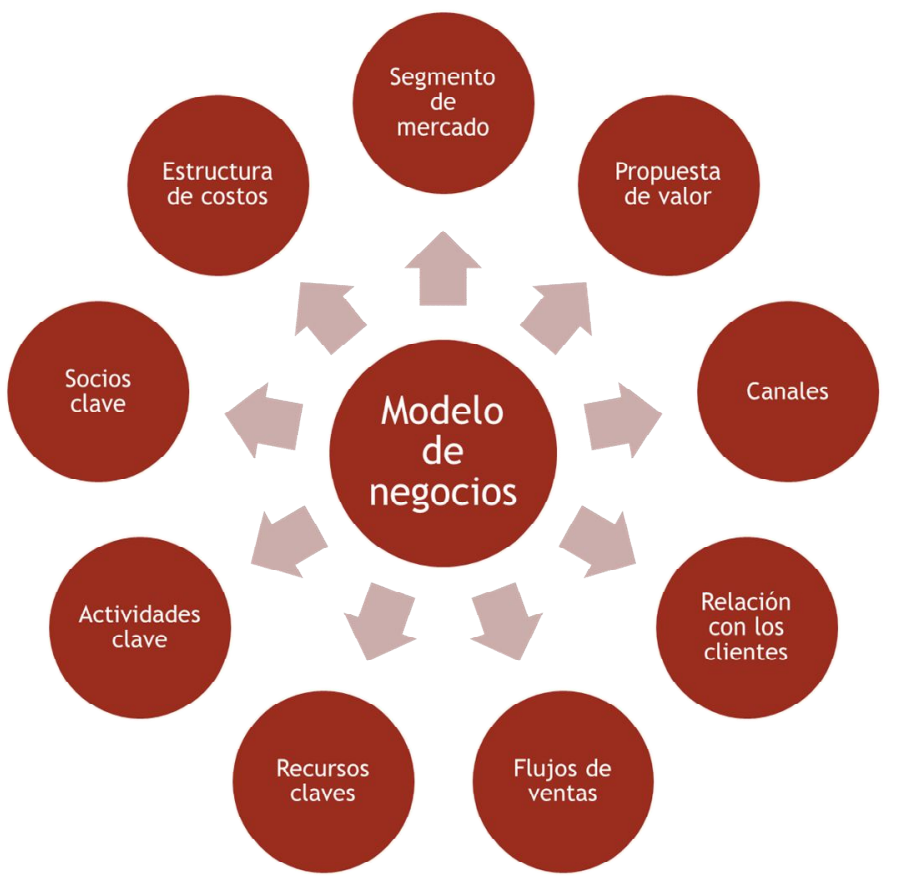

Fuente: Elaboración propia. 
Una revisión de la literatura arrojó diferentes artículos y trabajos sobre investigaciones publicadas que, a lo largo de los últimos años demuestran de manera clara la utilidad que tienen los análisis del BM, ya sean en una etapa temprana para iniciar con su diseño e implementación o en una etapa posterior cuando la mejor opción es una modificación o reinvención del $\mathrm{BM}$, principalmente en la industria manufacturera. Asimismo, se pudieron localizar métodos que abordan y analizan los modelos de negocios mayormente en ambientes industriales.

Para empezar, en un artículo publicado en el año de 2020 se indaga y presenta prácticas de gestión para crear y capturar valor en cierto tipo de BM, el cual es de tipo circular y se desarrolla en la industria manufacturera europea. Además, se destaca "la necesidad de concebir una perspectiva sistémica en la implementación de dichas prácticas” (Urbinati, Rosa, Sassanelli, Chiaroni, y Terzi, 2020: 1-11). Aquí comienza la idea de la importancia del BM, su desarrollo y seguimiento de una manera sistematizada, organizada y consciente del mismo, un trabajo de investigación reciente que afirma en la práctica lo que se menciona en la teoría. Asimismo, es importante resaltar cómo la implementación de prácticas a nivel gerencial puede apoyar el diseño del BM de las empresas manufactureras que lideran.

Así bien, se sugiere que una caracterización (con tendencia a diagnóstico y definición concreta y completa) del BM en la industria manufacturera con el fin de entender y clarificar los aspectos que están directamente involucrados en el proceso de creación de valor, pasando por los diferentes objetivos retos que tiene la empresa en sus operaciones y en su alcance en la cadena de suministro. De este modo se puede converger en la idea de que modificar el BM tiene resultados positivos ya sea modificando pocos componentes o aspectos mayores con un enfoque a los resultados mediante las nuevas tecnologías, siempre teniendo en cuenta que es importante conocer el panorama completo y de manera consciente para la aplicación de estrategias (Boffa y Maffei, 2019: 1360-1365).

También aplica lo anterior dicho cuando se estudia la industria de la manufactura aditiva con el enfoque del BM como herramienta. Es un tema relativamente nuevo y el efecto en general sobre el énfasis que se le da en los términos de operaciones son discutidos por los diferentes puntos de vista y efectos observables (Savolainen y Collan, 2020: 1-13).

México es uno de los principales representantes de la manufactura en Latinoamérica. Grandes compañías se sitúan o establecen sedes para la fabricación de productos en el país debido a su localización geográfica (cercanía con la importante zona de Norteamérica), fácil acceso por los bordes marítimos y la legislación y cuestiones políticas, sociales y económicas que favorecen la inversión extranjera, sin dejar de lado la calidad de la mano de obra (Vilchis y Aranda, 2015: 338-355).

Actualmente, en México también se estudia el BM desde los parámetros o factores determinantes que influyen en la innovación del mismo, es decir, se estudia el reto que significa generar un modelo de negocio exitoso en base de sus puntos clave. Todo lo anterior es con el fin de descubrir ciertos nichos que regulen y mejoren la ventaja competitiva de las organizaciones desde una continuidad o actualización del BM siempre teniendo en mente la innovación (Mejía y Sanchez, 2014: 107-132).

Aterrizando el tema al contexto de la región fronteriza de México con Estados Unidos, existe un artículo que describe algunos problemas o deficiencias en las operaciones de logística de la industria maquiladora, donde se desarrollan bajo modelos de negocios desarrollados o adaptados al entorno de específico local 
donde ocurre que la materia prima y los productos terminados deben recorrer grandes distancias en su cadena de suministros conectadas al estado y no se recibe la atención adecuada, por lo que es importante primero ser conscientes del problema o deficiencia existentes y como la mejora en el BM está directamente relacionado con problemas de la cadena de suministros (Restori, Aguirre, Ahumada, y Villalobos, 2004: 11651170).

La zona norte de México cuenta con una gran concentración de industrias maquiladoras destinadas a la manufactura de productos, dichas maquiladoras pertenecen en su mayoría a empresas extranjeras que invierten sus recursos en el territorio nacional para llevar a cabo sus operaciones, ya sea, para fabricar su producto final o generar componentes que se trasladan a otras fábricas o se exportan para ser terminadas fuera del país. Específicamente Ciudad Juárez, Chihuahua cuenta con un gran número de industrias de este tipo. Cabe señalar que existen de empresas que no terminan por completo el producto final que llega a los clientes las cuales son mayoría, aunque no por mucho, por la gran variedad de maquiladoras del sector (Bribiescas, Romero, y García, 2015: 69-79).

A su vez, las industrias maquiladoras de la región de Ciudad Juárez integran y adoptan metodologías y principios definidos por la manufactura esbelta tratando siempre de llegar a los objetivos propuestos por la matriz que es cumplir con los objetivos de productividad, calidad y costos, no dejando de lado que muy frecuentemente no se realizan las labores de investigación y diseño en la zona, sino solamente actividades de producción en masa (Bribiescas y Romero, 2014: 113-131).

La dinámica de la industria maquiladora está muy definida, sin embargo, se adapta al cambio y a las decisiones tomadas por la alta gerencia en función de ellas. La integración del análisis para la adaptación es una constante sin dejar de lado la identidad de las regiones, la cual por ejemplo es muy arraigada en Ciudad Juárez por su mano de obra (Salas, Limas, Jiménez, Jaramillo y Melgar, 2011: 30-35).

Así pues, el objetivo del presente trabajo de investigación es el conocer sobre el estatus actual del Modelo de Negocios (BM) en cuanto a su aplicación y características en la industria manufacturera de Ciudad Juárez, teniendo siempre en mente la diversidad de la misma y que los sectores son variados, por lo que en la medida de los posible se analizarán desde sus semejanzas. Asimismo, se define el método cualitativo de la entrevista estructurada realizada a expertos en el área de la industria antes mencionada, tanto directivos de empresas ubicadas en la región fronteriza como académicos con una carrera sólida con experiencia en la dirección de empresas e investigación de diferentes ámbitos de la administración. Al final se analizan los resultados para definir el contraste o semejanzas con la revisión bibliográfica para obtener conclusiones útiles para la posible mejora del BM en empresas de manufactura. Es decir, poder tomar como base las conclusiones para ser capaces de desarrollar y entregar de una propuesta con el análisis del BM de la industria maquiladora para su mejora continua con enfoques de la administración estratégica.

Todo lo anterior funciona como punto de partida, sentando las bases teóricas y antecedentes donde diversos trabajos de investigación sustentan la aplicación del mejoramiento, diagnostico o análisis del BM que a su vez pueden ser tomados como base e incluso tomar lecciones aprendidas para el desarrollo de la metodología y las diferentes etapas del mismo y trabajos posteriores. 


\section{Métodos}

El tema central BM, un tema de interés para administradores de empresas u organizaciones con fines de lucro, es un tema que normalmente es abordado desde el punto de vista cuantitativo. Siguiendo el método científico de manera rigurosa con el fin de obtener resultados, analizarlos y concluir sobre los mismos.

Sin embargo, dada la naturaleza del tema y sus vertientes el presente artículo adopta un método de investigación de índole cualitativo, es decir, se toma la decisión de abordarlo con un enfoque cualitativo en la viva voz de diferentes expertos en el tema de la industria manufacturera de Ciudad Juárez desde el punto de vista administrativo. Con el fin de hacer mano de la técnica de la entrevista para recopilar información. En los siguientes párrafos se indica la manera en la que es plantea el método de investigación

La técnica de la entrevista resulta relevante en la investigación científica, la principal razón es la flexibilidad tanto a la hora del diseño como en su ejecución. Asimismo, es un instrumento que resulta ser dinámico, ya que, puede indagar sobre fenómenos de diferente índole (social, ciencias exactas, información de tipo cualitativa) y también (Díaz-Bravo, Torruco-García, Martinez-Hernández, y Varela-Ruiz, 2013: 162-167). A su vez, por tratarse se puede aplicar a expertos Por dichas razones se optó por utilizar la herramienta mencionada.

Una de las ventajas de la técnica de la entrevista es la investigación y registro de "hechos no observables", que, de fuentes relevantes como lo son los expertos en cada materia revelan información. En específico, se elige la entrevista estructurada que consiste en la generar una serie de preguntas orientadas, realizarlas al sujeto en cuestión, escuchar y registrar las respuestas. Después, se redactan los resultados para hacer un análisis sin entrar en la libre interoperación. Si bien es de base cualitativa y de cierta manera subjetiva (exceptuando citas dadas por el o los entrevistados y confirmadas con la revisión bibliográfica) las coincidencias entre los entrevistados dan una guía de la realidad (Vargas, 2012: 119-139).

En el presente trabajo, se diseña la entrevista de tal manera que funcione como instrumento central que permita recopilar información relevante para la obtención de conclusiones contundentes en el tema y con la fundamentación. Dicha entrevista se aplicó a una población muestra compuesta por un pequeño grupo de siete funcionarios directivos y un experto consultor independiente en el sector de la industria maquiladora de Ciudad Juárez con experiencia en puestos administrativos de alta gerencia.

Se tomó como base también para la fase de la preparación de la entrevista (definición de objetivos, formulación de preguntas de acuerdo con la estructura establecida). Las preguntas utilizadas en el instrumento de estudio fueron las siguientes:

1. ¿Estás de acuerdo con estas definiciones? ¿Qué le podrías agregar o quitar?

2. ¿Qué tan importante es la definición de un BM para las grandes empresas, en este caso enfocándonos en la industria maquiladora de Ciudad Juárez?

3. De manera general, ¿ los directivos y líderes de las empresas, son conscientes de la importancia del modelo de negocios y conocen el porqué del mismo o solo se ejecuta?

4. ¿Hasta qué nivel es importante tener conciencia del BM (gerencial, coordinaciones, profesionales, ingeniería, operadores)?

5. ¿ ¿Se puede redefinir el BM desde las empresas que tienen varios años en operación o el cambio solo puede ser realizado desde la matriz? 
6. ¿Se puede definir un solo BM característico de las grandes empresas que funcionan en Ciudad Juárez?

7. ¿ ¿Ha impactado la llegada de la pandemia del COVID-19 de manera que las empresas modifiquen alguna cuestión del BM o no generó cambios significativos en ese sentido?

8. ¿Es similar el análisis de mercado de las empresas en ciudad Juárez?

9. ¿Hay productos que actualmente no manufacturemos en Ciudad Juárez y es posible que cualquier tipo de productos llegue a producirse en la ciudad?

10. ¿Hay procesos que actualmente no realicemos en Ciudad Juárez y es posible que cualquier tipo de proceso llegue a realizarse en la ciudad?

11. ¿Es importante un diagnóstico y mejora continua del BM o solo con la definición inicial y correcta ejecución es suficiente? ¿Por qué?

12. ¿Cuáles son los indicadores que muestran que el $\mathrm{BM}$ funciona?

13. Comentarios adicionales o conclusiones del tema

Para la segunda fase de las entrevistas programadas con algunos de los funcionarios directivos de la industria manufacturera en forma selectiva, la aplicación de las entrevistas fue por medio virtual, por la plataforma virtual de Microsoft Teams. Teniendo registro de las reuniones virtuales mediante la grabación de las sesiones para futuras referencias y un registro más claro y accesible.

Algunos de los objetivos deseados en la aplicación de las entrevistas con los directivos de las empresas de la industria manufacturera fue la identificación de la propuesta de valor de la industria de manufactura juarense, caracterización del BM en las maquiladoras, así como la identificación de los objetivos de la manufactura juarense. El registro de dicha aplicación se encuentra en la sección de resultados.

Posteriormente, se realiza una revisión literaria de trabajos de investigación meramente prácticos donde se lleguen a conclusiones relacionadas con el diagnóstico, mejoramiento, reformulación e innovación del BM. El objetivo de la revisión es la comparación de los resultados con la realidad de la industria maquiladora de la localidad. Contrastar o empatar resultados relacionados con el BM, análisis del mercado, producto y procesos.

\section{Resultados}

En la presente sección por su relevancia se presentan los resultados correspondientes a la entrevista aplicada al Dr. Francisco Arturo Bribiescas. quien es un experto consultor independiente, de profesión Ingeniero industrial, además de contar con una maestría en Administración y un Doctorado en Ciencias Administrativas, con más de 30 años de experiencia en empresas de manufactura, principalmente en 2 empresas Lear Corporation y Emerson en las cuales se desempeñaba como director de planta y gerente de operaciones. Experiencia en Benchmarking en plantas de Japón, China, Filipinas, Estados Unidos, Canadá y México. Trayectoria laboral que respalda el estatus de experto en el área de manufactura, para después convertirse en profesor e investigador en la Universidad Autónoma de Ciudad Juárez (UACJ).

La respuesta de la entrevista realizada al experto consultor independiente, se describen a continuación: 
1. ¿Estás de acuerdo con estas definiciones? ¿Qué le podrías agregar o quitar?

El concepto del BM para cualquier tipo de organización o empresa, debe contar con un modelo que lo lleve a las utilidades y a la rentabilidad. Siempre debe estar en constante revisión y si se requiere un hacer un rediseño por los constantes cambios y a los nuevos temas de interés, que lleva modificaciones de las mismas operaciones en función del avance tecnológico Se debe tomar en cuenta la estructura de costos, tipo de inversiones, procesos, utilización de energía entre otros temas. El BM se debe ir adaptando a las condiciones, no se puede quedar estático y la parte directiva es responsable de ello.

2. ¿Qué tan importante es la definición de un BM para las grandes empresas, en este caso enfocándonos en la industria maquiladora del sector manufactura en Ciudad Juárez?

El BM es el mapa del camino, todas las organizaciones de buen nivel están obligadas a tenerlo bien definido. Implementándolo y diseñándolo, incorporando temas de interés actuales, para determinar las estrategias que se utilizarían para llevarlo a cabo.

3. De manera general, ¿̇os directivos y líderes de las empresas, son conscientes de la importancia del BM y conocen el porqué del mismo o solo se ejecuta?

Es muy aventurado afirmar algo al respecto, se debe tener una base de datos y una recopilación de datos estructurada. Sin embargo, en la experiencia en la industria maquiladora de la localidad no todos los directivos tienen claro el concepto del BM y es responsabilidad de los líderes mantener un nivel de actualización en términos gerenciales y de operaciones. Empresas con un BM poco definido es probable que sus operaciones y existencia tenga una vigencia menor a las que si lo tienen, entonces, la búsqueda de la definición del BM es responsabilidad de la alta gerencia.

4. ¿Hasta qué nivel es importante tener conciencia del BM (gerencial, coordinaciones, profesionales, ingeniería, operadores)?

El BM es algo que se debe compartir con cada parte de la estructura organizacional (normalmente estructura piramidal), pero cuando hay poca claridad en el BM, se dificulta el manejo de la información, así como el mensaje que se quiere dar para transmitir e inyectar la importancia de los objetivos de las empresas, incluyendo el constante replanteo de las estrategias tomadas desde el monitoreo del mercado y la situación actual en el mundo empresarial.

5. ¿Se puede redefinir el BM desde las empresas que tienen varios años en operación o el cambio solo puede ser realizado desde la matriz?

Existen empresas de la industria maquiladora en Ciudad Juárez que cuenta con autonomía y libertad para tomar decisiones, sin embargo, la mayoría de las compañías son manejadas por las matrices que se encuentran en diferentes países de primer mundo. Existe una tendencia a la descentralización, pero es dominante la administración de organizaciones que centralizan el poder. Se debe trabajar para lograr que las restricciones en la toma de decisiones permitan mayor libertan en aras del mejoramiento del estatus de la empresa de manera significativa, no solo en los métricos convencionales de rendimiento y eficiencia. 
6. ¿ ¿Se puede definir un solo BM característico de las grandes empresas que funcionan en Ciudad Juárez?

Es difícil definir un solo BM característico, existe una gran variedad de sectores en los que se divide la industria manufacturera. En Ciudad Juárez se pueden dividir en 8 grupos, como lo son el sector de arneses, el sector automotriz, el de costura, productos eléctricos y electrónicos, industria de materiales de uso médico, la industria metal mecánica y la del moldeo de plásticos. Depende de cada sector como evolucionar, por ejemplo a nivel global, la industria automotriz cuanta con planes a varios años a futuro, tal es el caso de Toyota que planea generar la mitad de sus ventas a partir de vehículos eléctricos (Levin, 2020), o BMW que tiene como objetivo incrementar en un $50 \%$ la producción de su línea de vehículos eléctricos (Carey y Fenton, 2021), a su vez GM aumentará su inversión para la producción de 30 modelos eléctricos en 2025 (Auto, 2020) y por su parte Ford invertirá 11 mil millones de dólares para la manufactura de 40 vehículos eléctricos en 2022 (Reuters, 2018). Cada una de estas decisiones son inherentes al BM y nos afecta en la producción en la localidad directa o indirectamente. Donde las empresas deben tener al menos una planeación estratégica del negocio a 5 años.

7. ¿ ¿Ha impactado la llegada de la pandemia del COVID-19 de manera que las empresas modifiquen alguna cuestión del BM o no generó cambios significativos en ese sentido?

Los impactos de las crisis económicas en los $\mathrm{BM}$ son claros e inminentes y ahora con la pandemia del COVID-19 no es la excepción.
Comenzando en la manera que evolucionó el trabajo remoto de cierta parte del personal trabajador de la industria maquiladora. Ahora bien, los procesos remotos no son una realidad total actual, sin embargo, en el presente se puede estar fraguando procesos tecnológicos de este tipo donde el reto es el ajuste de materiales y procesos. Por lo pronto las afectaciones golpearon en mayor o menor medida a las empresas de todos los tamaños y establecidas en cualquier parte del mundo, pero se están buscando soluciones a problemas de este tipo para evitar posibles repercusiones similares en el futuro. Lo anterior aplica para las empresas maquiladoras y los diferentes componentes de la cadena de suministros y distribución de materiales a los consumidores finales.

8. ¿Es similar el análisis de mercado de las empresas en ciudad Juárez?

Actualmente, en la región de Ciudad Juárez se localizan de 450 a 500 empresas que se dedican a la manufactura de productos. La gran mayoría de ellas cuentan con una estrategia global de manufactura, con objetivos encausados al crecimiento y a la rentabilidad. El análisis del mercado, así como las estrategias están bien definidas y ciertamente tienen similitudes, algunas en mayor o menor medida, incluso dependiendo del sector. Para empezar, se toma la ventaja de la ubicación geográfica, ventajas de la relación de calidad y costo de mano de obra, entre otros aspectos. Es importante mencionar que el análisis de mercado es similar y es igual de importante las medianas empresas con las grandes que operan en la ciudad tanto las que ofrecen productos como las de servicios. 
9. ¿Hay productos que actualmente no manufacturamos en Ciudad Juárez y es posible que cualquier tipo de productos llegue a producirse en la ciudad?

Actualmente se produce una gran variedad de productos en Ciudad Juárez, sin embargo, por la misma diversidad sería imposible manufacturar exactamente todos, pero la capacidad de la mano de obra, instalaciones y procesos en la ciudad es bastante para producir una gran cantidad adicional. Pero, es una realidad que algunas empresas no optan por la producción alta tecnología, alta medicina o software especializado. La facilidad de la obtención de mano de obra no es compatible con la necesaria para la producción con dichas características, la base humana y la base tecnología, no solo en la ciudad o la región sino en el país.

10. ¿Hay procesos que actualmente no realicemos en Ciudad Juárez y es posible que cualquier tipo de proceso llegue a realizarse en la ciudad?

$\mathrm{Al}$ igual que el punto anterior, los argumentos son igualmente válidos. Gente competitiva, preparada y con bases que tengas la especialización necesaria para la implementación de nuevos procesos de alta tecnología, incursión en la instalación de centros de diseño e innovación. La relación entre los productos y procesos de alta tecnología es muy estrecha.

11. ¿Es importante un diagnóstico y mejora continua del BM o solo con la definición inicial y correcta ejecución es suficiente? ¿Por qué?

Sí, es importante. Sin embargo, existen métodos y herramientas para soportar y afianzar el BM. Es muy importante la elección de estas herramientas y la buena gestión está basada en la determinación de las herramientas adecuadas a utilizar en las empresas para las operaciones con el fin de generar valor. En la actualidad está muy de boga el avance de la tecnología con la automatización y el mercado es dinámico, por tal razón es primordial el mantenimiento del BM con las herramientas correctas, tanto para los procesos como para la dirección de las personas. También, es necesario ver si el BM funciona, puede ser un buen diseño, pero podría no estar funcionando, es necesario evaluarlo y ajustarlo para su mejor rendimiento. En un periodo aceptable de tiempo es incluso recomendable rediseñar el BM.

12. ¿Cuáles son los indicadores que muestran que el $\mathrm{BM}$ funciona?

Existen los "Key Performance Indicators” (KPI) operativos que indican convencionalmente los niveles de calidad, producción, eficiencia. Pero más allá de esos indicadores existen otros muy interesantes sin importar si se tiene alta automatización o no, como lo es las horas estándar por pie cuadrado, es decir, relaciona espacio con capacidad para producir piezas en un periodo de tiempo, en otras palabras, un indicador de espacio productivo y que no es muy común y muy útil para reflejar la situación del $\mathrm{BM}$ en las operaciones. Otros podrían ser la cantidad de patentes, indicadores de innovación o costos de la mala calidad (COPQ). Y otro sería hacer benchmarking de plantas de alto nivel.

Ahora bien, un trabajo de investigación realizado en 2015 arrojó una herramienta práctica que ayuda en los cálculos relacionados a los costos con diferentes funciones, bajo la reformulación de un BM preestablecido. Se evaluó bajo un análisis de 
sensibilidad, lo cual lleva a una metodología bien fundamentada. Incluso se propuso la directa implementación en los procesos de producción relacionados a la manufactura aditiva y toda la nueva tecnología implicada (Schröder, Falk, y Schmitt, 2015: 311-316). Este es un ejemplo donde se desarrolló un nuevo BM y se trata de evaluar la viabilidad y posible efectividad en su implementación.

En una investigación distinta se revisa la manera del diseño del BM tomando en cuenta la flexibilidad en la manufactura. Se analiza la tendencia de la creciente diversidad de los BM y sus diferentes enfoques, que, cabe mencionar son cambiantes y dinámicos en cortos periodos de tiempo. Lo anterior entra en un tema de gran interés para los administradores desde un punto de vista empresarial evolutivo. Específicamente, el artículo presenta implicaciones importantes en el sentido gerencial para que los encargados de las operaciones mejoren el desempeño de la empresa con el hecho fundamental del rediseño del BM. Nuevamente considerando seriamente las nuevas tecnologías haciendo énfasis en las tecnologías de la información que son un medio para la creación eficiente de valor (Wei, Song, y Wang, 2017: 87-97).

Continuando con el tema de las nuevas tecnologías, otro trabajo, presenta de manera puntual las oportunidades y desafíos al que se enfrentan las empresas, pero que con el enfoque correcto se pueden aprovechar para un acelerado crecimiento teniendo la herramienta del BM bien diseñado e implementado, adaptado a industrias de productos y servicios haciendo su distinción donde sea necesaria. Al final se hace hincapié en el impacto positivo o negativo del BM dirigido hacia las empresas tecnológicas (Paiola y Gebauer, 2020: 245-264). Cabe mencionar que el rediseño no solo incluye seguir las reglas puestas por las grandes empresas exitosas sino adaptarlo a las necesidades propias del giro de la empresa, incluso llegando a crear novedosos métodos para diseñar y aplicar el BM.

También, un artículo más analiza más allá de las grandes empresas, es decir, las pequeñas y medianas empresas de manufactura y su relación con la reciente introducción de la industria 4.0 y como las mismas se debe adaptar en el sentido del BM. La investigación fue realizada en empresas alemanas y demuestra un cambio gradual y lógico en la manera que crean valor, de cierta manera de forma obligada. Abarcando desde procesos internos, manera de aplicarlos hasta ciertos puntos de la cadena de suministros. Se debe aprovechar todos los recursos de las nuevas tecnologías como lo son la digitalización de los procesos, la manufactura asistida y la conectividad para asegurar la utilizad y la sobrevivencia a la vez que se ofrecen productos y servicios atractivos en diferentes sentidos (Müller, Buliga, y Voigt, 2018: 2-17).

Finalmente, con el fin de realizar la comparación con un caso más local de análisis del BM tenemos un trabajo doctoral, que hace un análisis extenso sobre una "Propuesta metodológica para el desarrollo de negocios en la industria maquiladora de autopartes en $\mathrm{Cd}$. Juárez”. Al final se llegan a diferentes conclusiones, de lo cual se puede obtener la importancia del diagnóstico del mercado, análisis de producto y procesos que son factores de la generación de la ventaja competitiva (Acosta, 2019: 1-172). Aunque no se mencioné explícitamente el BM como objeto de estudio, es justamente la importancia de analizar todos estos factores que llevan o no a una empresa al éxito mediante la correcta implementación de una herramienta para capturar y crear valor. 


\section{Conclusiones}

El constante monitoreo del BM y la confirmación de su aplicabilidad en la actualidad en la que se vive es tan importante como los resultados reflejados en los principales indicadores de eficiencia, producción y costos, ya que directamente impacta a los mismos y a algunas otras áreas relevantes en las operaciones de las empresas.

Asimismo, el diagnóstico y mejoramiento del BM ayuda a las grandes empresas de la industria maquiladora en Ciudad Juárez a mantener su vigencia y la adaptabilidad. Es primordial para la dinámica de la economía y las operaciones hoy en día, tener en cuenta el constante cambio de las generaciones y el avance de la tecnología en los productos y procesos, así como los factores que afectan la demanda y por ende el negocio.

Por otro lado, la diversidad de las empresas de la industria maquiladora en Ciudad Juárez no permite la caracterización de un BM en común. Sin embargo, si se tienen similitudes, es mayormente producción en masa de partes a ensamblar en alguna otra planta, dejando rezagado el diseño y la manufactura de productos para el cliente final. Aunque sea cual sea el sector de la industria se exporta y se aprovechan las ventajas de la ubicación geográfica.

Además, la importancia del BM es comparable tanto como en grandes empresas ubicadas en otras partes del mundo, la tecnología juega un papel importante en cada una de ellas. La innovación en el BM es un indicador que puede predecir el futuro éxito de las empresas.

Finalmente, se tienen similitudes en productos y procesos desarrollados en la región, actualmente no tienden ser de alta tecnología, sin embargo, se tiene el potencial y para mejorar en ese sentido se debe trabajar en un alto grado de formación de la mano de obra e incluir procesos especializados acorde a un BM pensado estratégicamente de manera integral con los objetivos de las empresas de manera local y global.

\section{Referencias}

Acosta, L. (2019). Propuesta metodológica para el desarrollo de negocios en la industria maquiladora de autopartes en Cd. Juárez. Secretaría de Investigación y Posgrado - Universidad Autonóma de Chihuahua, 1172.

Auto, I. (2020, Noviembre 19). GM to boost spending on electric vehicles, plans 30 models by 2025. Retrieved from The Washington Post.

https://www.washingtonpost.com/business/economy/g m-to-boost-spending-on-electric-vehicles-plans-30models-by-2025/2020/11/19/15d68534-2a61-11eb92b7-6ef17b3fe3b4_story.html

Boffa, E., y Maffei, A. (2019). A descriptive framework to characterize the manufacturing domain in the context of Business Models. Procedia CIRP, 81(1), 1360-1365.

Bribiescas, F. A., y Romero, I. F. (2014). Gestión de certificación de calidad como factor de competitividad en le sector industrial de manufactura, en la región transfronteriza Cd. Juárez, Chih., México, El Paso, Texas, USA. Revista internacional administración \& finanzas, 7(1), 113-131.

Bribiescas, F. A., Romero, I. F., y García, E. (2015). Turismo industrial: oportunidades y retos dentro del sector de manufactura en Ciudad Juárez. óesis: Revista de Ciencias Sociales y Humanidades, 24(47), 69-79.

Carey, N., y Fenton, S. (2021, Enero 15). BMW aims to double fully-electric vehicle sales in 2021. Reuters. https://www.reuters.com/article/autos-bmw-electricidINKBN29K25A 
Chesbrough, H. (2010). Business model innovation: opportunities and barriers. Long range planning, 43(23), 354-363.

Díaz-Bravo, L., Torruco-García, U., MartinezHernández, M., y Varela-Ruiz, M. (2013). La entrevista, recurso flexible y dinámico. Investigación en educación médica, 2(7), 162-167.

Levin, T. (2020, Enero 28). All the things carmakers say they'll accomplish with their future electric vehicles between now and 2030. Business Insider. https://www.businessinsider.com/promises-carmakershave-made-about-their-future-electric-vehicles-20201 ? $\mathrm{r}=\mathrm{MX} \& \mathrm{IR}=\mathrm{T}$

Mejía, J., y Sanchez, J. (2014). Factores determinantes de la innovación del modelo de negocios en la creación de ventaja competitiva. Revista del Centro de Investigación. Universidad La Salle, 11(42), 107-132.

Morris, L. (2010). Business model innovation the strategy of business breakthroughs. International Journal of Innovation Science, 1(4), 191-204.

Müller, J. M., Buliga, O., y Voigt, K.-I. (2018). Fortune favors the prepared: How SMEs approach business model innovations in Industry 4.0. Technological Forecasting and Social Change, 132(1), 2-17.

Paiola, M., y Gebauer, H. (2020). Internet of things technologies, digital servitization and business model innovation in BtoB manufacturing firms. Industrial Marketing Management, 89(1), 245-264.

Restori, M., Aguirre, F., Ahumada, O., y Villalobos, R. (2004). Improvement of Transportation Practices in the Maquiladora Industry. IIE Annual Conference. Proceedings, 1165-1170.
Reuters. (2018, Enero 15). Ford plans $\$ 11$ billion investment, 40 electrified vehicles by 2022. CNBC. https:/www.cnbc.com/2018/01/15/ford-plans-11billion-investment-in-electric-vehicles-by-2022.html

Salas, J. F., Limas, M. L., Jiménez, B., Jaramillo, C., y Melgar, J. (2011). La motivación y la disposición al cambio de los trabajadores en mandos medios de la industria maquiladora de Ciudad Juárez. NovaRua Revista Universitaria de Administración, 2(3), 30-35.

Savolainen, J., y Collan, M. (2020). How additive manufacturing technology changes business models?review of literature. Additive manufacturing, 32(1), 113.

Schröder, M., Falk, B., y Schmitt, R. (2015). Evaluation of cost structures of additive manufacturing processes using a new business model. Procedia Cirp, 30(1), 311-316.

Teece, D. (2010). Business models, business strategy and innovation. Long range planning, 43(2-3), 172194.

Urbinati, A., Rosa, P., Sassanelli, C., Chiaroni, D., y Terzi, S. (2020). Circular business models in the European manufacturing industry: A multiple case study analysis. Journal of cleaner production, 274(1), $1-11$.

Vargas, I. (2012). La entrevista en la investigación cualitativa: nuevas tendencias y retos. Calidad en la educación superior, 3(1), 119-139.

Vilchis, A., y Aranda, E. (2015). Transformación del mercado laboral profesional en la industria maquiladora en Ciudad Juárez, Chihuahua en el 2010. European Scientific Journal, 11(34), 338-355. 
Wei, Z., Song, X., y Wang, D. (2017). Manufacturing flexibility, business model design, and firm performance. International Journal of Production Economics, 193(1), 87-97.

Zott, C., y Amit, R. (2010). Business model design: An activity system perspective. Long range planning, 43(2-3), 216-226.

Zott, C., Amit, R., y Lorenzo, M. (2010). The business model: Theoretical roots, recent developments, and future research. IESE Research Papers, 3(4), 1-43. 